\title{
A propósito da Vigilância em Saúde do Trabalhador
}

\author{
Workers' Health Surveillance
}

Jor ge Mesquita Huet Machado 1

\begin{abstract}
This article presents a discussion about the context of the Surveillance in the area of Workers Health (SWH). It is assumed to be a transdisciplinary social praxis in which a variety of social actors are organized, making part of a heterodox and constructivist process. The last decade Brazilian institutional and histo rical evolution is focused. So as the constitution of SWH netwo rks in a field marked by contradictions between so cial values such as the health as a principle and the production demands.

Key words Surveillance in Workers Health, Workers Health, Transdisciplinary actions
\end{abstract}

Resumo Este texto apresenta uma discussão co n textualizada das ações de Vi gilância em Saúde do Tra balhador (VST), na qualidade de uma práxis social transdisciplinar em que distintos atores se organizam em um processo heterodoxo e construtivista. Destaca-se o momento histórico institu cional vivido pelo Brasil nas últimas décadas e a consti tuição de redes de VST a partir de objetos priorizados em um campo de contradições entre a saúde e a produção como valores sociais. Palavras-ch ave Vigilância em Saúde do Trabalhador, Saúde do Trabalhador, Transdisciplinaridade

1 Coordenação de Saúde

do Trabalhador,

DIREH/Fiocruz.

Pavilhão Figueiredo

Va s con celos, sala 301, Av. Brasil 4365,

Rio de Jan ei ro RJ.

jorgemhm@procc.fiocruz.br 


\section{Introdução}

As questões aqui apre s en $\mathrm{t}$ adas corre s pon dem a algumas inquietações referentes à prática de Vigilância em Saúde do Trabalhador (VST), desenvolvida no Brasil nestes últimos vinte anos. Foi a partir de 1994 que, no âmbito do SUS, houve um movimen to de generalização de experiências estaduais iniciadas na década anteri or e um processo de municipalização das ações de saúde do trabalhador decorrente daquelas experiências estaduais mais consolidadas. A II Conferência Nacional de Saúde do Trabalhador esteve marcada por esse con texto de significativa ampliação do número de programas, cen tros regionais, núcl eos e coorden ações de saúde do trabalhador e inclusive dos marcos legais da áre a.

A prática de VST é considerada, no entanto, fugaz e regionalizada (Lacaz et al., 2002). Fugaz, em virtu de do grau de instabilidade das experiências e da sua limitada resistência às f reqüentes mudanças internas - principalmente no âmbi to municipal -, que inviabilizam o desenvolvimen to de muitas iniciativas, algumas das quais nem ch egam a decolar. E regionalizada, por se concentrar principalmente em São Paulo - devi do à influência em sua ori gem do movimen to sindical e de sanitaristas da Sec retaria de Estado de Saúde - e em algumas regiões no Sudestee Sul, bem como na Bahia pelo papel estruturan te exercido pelo Cen tro Est adual de Sa ú de do Trabalhador (CESAT).

Nos últimos três anos, com a implantação da Rede Nacional de Atenção In tegral à Saúde do Trabalhador (Renast), há uma tentativa de pro ta gonismo maior do SUS na formulação de uma política clara de expansão das ações em saú dedo trabalhador.

É necessário mencionar ainda que as ações de saúde do trabalhador passam por um processo de amadu recimen to e difusão, derivado da con fig u ração do SUS e de suas relações com o movimen to sindical, com instâncias do Ministério da Previdência, do Trabalho e do Meio Ambiente, com setores empresariais, corporações técnicas, aparatos formadores técnicocientíficos e agências de regulação envolvidas de forma mais próxima na interação da saúde com o trabalho (Mach ado, 2003).

Em termos gerais, pode constatar-se que a implantação de ações de VST de s envo lvidas no s etor saúde por gru pos institucionais localizados em vários pon tos do Brasil, com o grau de con cen tração referi do anteriormente, resultou em uma gama de ex periências distintas. As diferenças observadas refletem as potencialidades regionais, que giram em torno da força e qualidade da organização dos trabalhadores quanto às questões de saúde. Em termos institucionais, dependem das políticas regionais e da estrutu ra organizacional, da capaci d ade instalada, da qualidade dos profissionais envolvidos e de influências advindas das instituições acadêmicas (Lacaz et al., 2002; Machado, 1996).

Ca be de st acarnesse cen á rio de ex periências h eterogêneas algumas caracterís ticas comuns e es truturantes do modelo de VST desenvo lvi do no país e como são constituídas redes de ação em que operam vários níveis de VST por diversos atore s, em um processo de integração organizado pelos objetos con cretos sobre os quais se tecem teias de ligações interinstitucionais.

\section{Características estruturan tes da VST}

\section{A relação interinstitucional}

Ao recortarmos a vigilância ten do como foco o campo da saúde do trabalhador, def ron tamo-nos, na prática, com o problema da integração. Verifica-se intensa multiplicidade temática, abrangen do diversas disciplinas, que não redundam necessariamente na elaboração de uma política pública, radicalmen te inters etorial e a rticulada com outros âmbi tos da vigilância.

A maioria das insti tuições se ressente da influência de con cepções técnicas e de recursos humanos form ados seg u n douma lógica ac ad êmica compartim entar em faculdades e departamentos que dificulta a integração entre as disciplinas. Verificam-se, apenas, algumas ilhas interdisciplinares volt adas para a Saú de Col etiva. Mesmo nessas ilhas, atri bui-se pouca ênfase à saúde dos trabalhadores, tornando inadequ ada e insufici en te aformação de recu rsos humanos. As barrei ras disciplinares mais uma vez se in terpõem diante da racionalidade necessária para uma ação transversal em saúde do trabalhador. A reprodução de uma estrutura disciplinar organizada secularmen te faz com que estejam consolidados poderes e preconceitos, acarretando, do ponto de vista do cotidiano acadêmico pouca disponibilidade para o diálogo. Tal lógica termina por fundamentar a reprodução do saber e a perpetuação de práticas corpora tivas.

O processo de su peração desse cenário institucional é ainda incipien te e vem permeado 
por uma série de conflitos, alguns deles de tipo corporativo mais relacionados a disputas no campo das práticas institucionais ainda não harmonizadas pós-Constituição de 1988. Assim, auditores fiscais do Ministério de Trabalho e sanitaristas do SUS, voltados para as ações de saúde do trabalhador, vivem em conflitona maioria dos Estados brasileiros, on de se realizam, com maior ênfase, prá ticas nesse campo.

\section{A transdisciplinaridade}

A Vigilância em Saúde do Trabalhador como uma área de promoção da saúde requer pela natureza de suas práticas uma compreensão transdisciplinar e uma ação transversal inter e intra-setorial.

Essa exigência de uma formulação con ceitual que acolha e condicione as ações de VST permite avançar na reflexão lógica para além das disciplinas e arcabouços interdisciplinares fundamentados em esferas de dominância de sta ou daqu ela disciplina. Sob uma ótica de integração transdisciplinar, os componen tes básicos - sociais, técnicos e epidemiológi cos - da abordagem interdisciplinar da saúde do trabalh ador se articulam harm onicam en te de form a a que não mais prevaleçam vieses tecnicistas, sociológicos ou sanitári os, quantitativos ou qu alitativos. Somam-se a isso processos de construção de redes intra- e interin stitu cionais com vistas à preservação da saúde dos trabalhadores, ten do como estratégia a exclusão dos condicionantes de desgaste ou, ao menos, a redução de seus impactos.

A falta de uma base conceitual capaz de a justar as ações transversais, aliada a hierarqu izações que não correspon dem aos papéis possíveis de serem desempenhados por uma ou outra instituição isoladamente, constitui um dos empecilhos fundamentais para a objetivação das ações de VST. A proposta do SUS, em sua formulação da Sa ú de do Trabalhador, estabelece uma centralidade disciplinar da epidemiologia, configurando um saber dogmático freqüente na área de saúde. Por sua ve z, no âmbito do Ministério do Trabalho, o arcabouço in terdisciplinar da saúde do trabalhador acaba reduzindo-se à submissão das disciplinas a possibilidade de negociação no âmbi to das relações de trabalho e do engessamen to de sua prática por um mosaico inacabado de referenciais normativos. Essa disputa teó rica é muitas vezes intuitiva e, sobretu do, condicionada pelos próprios micropoderes exis ten tes nas insti- tuições de referência. Isso inclui, de forma perversa, os corpora tivismos que se auto - reproduzem como fim de sua ação institucional, con stituin do-se num campo fech ado de negócios e saberes.

O desafio de superar o que poderia qualificar-se como uma tendência de inércia conflitiva é bastante atual e só acon tecerá se enfrentarmos, conceitualmen te e de forma consciente, as bases de nossas práticas cotidianas, em suas dimensões ex ternas aos pró prios setores, como a implem entação de políticas públicas e de açõe s situadas num con tex to social mais amplo.

Con cei tos derivados do campo da saúde do trabalhador e das relações do trabalho são fundamentais para o entendimento e para as ações con c retas desenvo lvidas nos últimos anos nas insti tuições e na academia por departamentos multiprofissionais e interdisciplinares. As bases da epidemiologia social da América Latina (Lau rell \& Nori ega, 1989) e da ergon omia fra ncesa de Alain Wisner(1987), aplicadas ao mu ndo do trabalho, trouxeram avanços e repre sentam fundamen tos essenciais para a intervenção sanitária no campo das relações en tre a saúde e o trabalho. En tretanto, con cei tos como carga e a tividade, oriundos de disciplinas e arranjos ainda interdisciplinares, não dão conta da dimensão socioeconômica como um condicionante macroe s trutu ral da relação saúde - trabalho, trazen do tensões aos gru pos que de s envolvem suas práticas institucionais. Diante desse impasse, é necessária a adoção de con cei tos que conciliem o arcabouço multidisciplinar em uma pers pectiva transdisciplinar, de modo que tais con ceitos sejam com partilh ados por diversas disciplinas.

A abordagem dos condicionantes sóciotécnicos representa um el em en to de qualidade e um componente fundamental para o entendimen to da VST como estra té gia transdisciplinar de atuação con struída a partir dos obj etos, ou seja, a partir da ob s ervação e intervenção na relação en tre múltiplos processos de trabalho e saúde, con si derando suas especiais caracter ís ticas con strutivistas e histónicas.

\section{A construção de processos articulados de intervenção}

A análise da relação da saúde com o proce sso de trabalho - con tex tualizando as situações de risco técnico com as relações de trabalho e com as formas de resistência e de sgas te da saúde dos trabalh adores - cons titui o obj eto gen érico de todas os processos de vigilância em saú- 
de do trabalhador (Machado, 1996). Esse obj eto está inseri do como um núcleo de uma célula com várias esferas de raios distintos e ainda com con exões entre as diversas esferas con cêntricas ao redor do núcleo, conforman do uma rede de vigilância (Figura 1). Essa representação sintetiza o campo de ação da VST com suas várias formas de intervenção e suas distintas responsabilidades e relações.

No núcl eo, destacam-se os nucléolos, configurando os trabalhadores e o ambiente de trabalho, e uma divisão en tre os hemis férios do patron a to e dos trabalhadores. Aí reside o foco das ações de VST, estabeleci do a partir das contradições en tre a produção e a saúde dos trabalhadores. Constitui um espaço da ação individual e coletiva de resistência dos trabalhadore s diantedo processo de gestão do trabalho exercido pelas forças de comando no interior das em presas que conformam estraté gias de domínio da organização do trabalho e, conseqüentemente, das tecnologias adotadas ou vigentes nos processos de trabalho.

Ao nível da membrana que separa o núcl eo do restante da célula, operam-se as ações dos s erviços de saúde e segurança do trabalho das em presas e dos sindicatos, no sentido de dar maior ou men or permeabilidade às forças decorren tes dos campos esféricos de fora do núcl eo.

Um pri m ei ro ti po de VST é exercido no próprio interior dos núcleos pelos atores aí presentes, trabalhadores e seus representantes (cipistas e sindicato s), $\mathrm{p}$ a trões e seus repre s entantes (ch efias) e técnicos dos serviços especializados, tradicionalmente chamados de Serviços Especializados em Segurança e Medicina do Trabalho (SESMT).

A prim ei ra camad a, form ada por órgãos do poder exec utivo, tem como característica básica o alto grau de instabilidade. Volatiliza-se se não vem acompanhada de outras para constituir uma rede de apoio que consolide as ações decorren tes deste âmbito de intervenção. Os núdeos correspondem aos cen tros de referência, aos serviços e às coordenações do SUS, que desempenham ações de intervenção e discussão nos locais de trabalho e também a outras instituições que podem ou não estar articuladas ao SUS, como as Delegacias Regionais do Trabalho, Secretarias de Meio Ambiente e da

Figura 1

Rede de vi gilância em saúde do trabalhador.

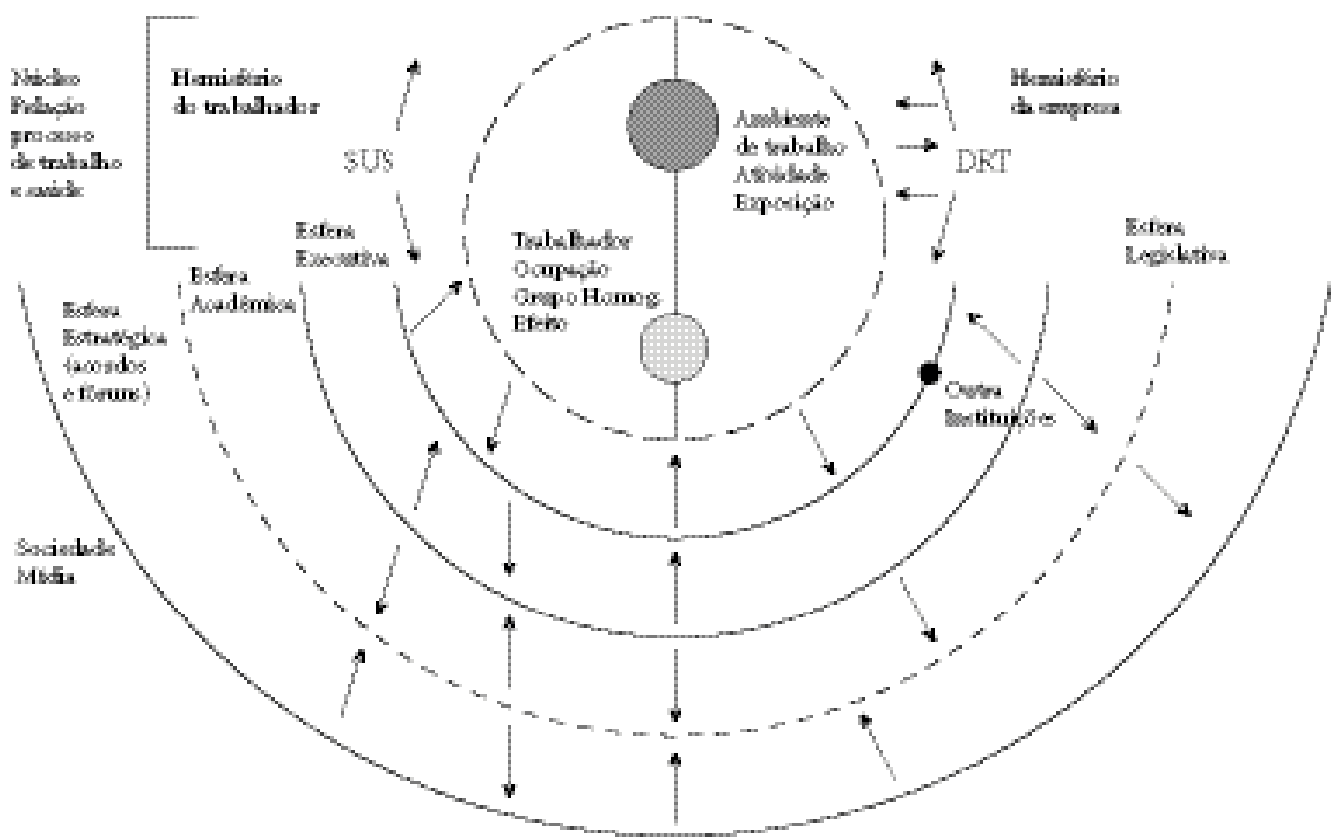

Modificado de Porto MSF, Mach ado JMH \& Freitas, CM 2000. 
Agricultura, Ministérios Públi cos e instâncias da Previdência Social, que executam uma ação institucional de VST, ou seja, um segundo tipo de VST.

Uma segunda camada é representada por um segmen to estratégico estrutura $n$ te que é o a p a relho form ador e de formulação e reprodução do conhecimento. É um lócus do saber, em que se disputam as bases fundamentais das ações e se produzem técnicos e discursos com lógicas que auxiliam as instituições exec utivas em suas ações. As repercussões das formulações transdisciplinares, interdisciplinares e disciplinares ado tadas por departamentos, em presas e insti tuições se tornam visíveis e palpáveis nas contendas por entendimentos e conceitos que fazem evo luir para um lado ou outro a delimitação dos hemisférios do núcl eo que repres enta o maior ou men or desgas te do trabalhador no exercício de seu trabalho. Pode ser vis to como compon en te epistemológi co da vigilância, ou mesmo de vigilância epistemológica, e se configura em um terceiro tipo de VST.

A próxima camada é constituída por elementos estratégicos na con s trução da rede trabecular e das relações entre as instâncias da mesma camad a. Exemplos de seus mecanismos são os Fóruns de Acordos, as Câmaras Técnicas, os Conselhos e outras formas de aglutinação inter e intra-institucionais. A sua função é tecer a rede, que permite dar flexibilidade e resistência ao processo de VST, tornando-se o lócus das negociações, dos conflitos e da con s trução de consensos e avanços estruturantes que vão se consolidar na camada a seguir. Essa é a camada da síntese de acordos e formalizações em termos legais, sendo consolidada por pactos jurídicos institucionais, estabelecendo avanços no teci dosocial democráti co e solidificando os progressos conquistados pelo proce sso de VS T. Essas duas camadas são mui to próximas e se tocam constantemente, uma estrutura n do a outra em uma figura en trelaçada, como uma coroa de espinhos. Deve-se destacar aqui o papel do MTE, pois sua ação nesse campo de negociações é exemplar e fundamental pela sua tradição de regulação das condições de trabalho. Nesse sentido, são ilus trativos acordos como o da CNP-Benzen o, o dos Vasos sob Pressão, o da Comissão da Resolução 174 e o das Prensas Injetoras, en tre outras experiências de regulação das condições do trabalho em situações de alto risco. Mais recentemente, cabe também destacar as ações parlamentares nas discussões de marcos legais, sejam eles nacio- nais ou regionais, como no caso da Comissão de Di rei tos Humanos da Câmara e de As sembléias Legislativas de Estados e Municípios (Cime, 2000; Lima 1993).

Esses pólos de con s ti tuição de redes não são únicos nem primários, entretanto são fundamentais, pois consolidam processos de negociação aproximan do pólos antagônicos, instituições e atores dispersos. Representam espaços concretos de formulação e acompanhamen to da ação transdisciplinar em um ajuste permanente em que as forças atuantes possam ter um fundamen to lógi co comum.

Constituindoa camada mais externa, como uma mem brana celular ex terna que delimita a rede de VST, a pre senta-se a instância do senso comum e de disputa do discurso na sociedade, em que os con s ensos se tornam senso comum, sen do estes bons sensos ou não. É o campo em que se defin emos diferentes graus de perigo e de risco que a sociedade permite em um determinado momen to e território. Expressa também as situações de exclusão e de inclusão que estão va l en doe quais serão os ti pos de "núdeos" (processos de trabalho) possíveis e desejáveis.

A difusão da informação e o direi to do saber são estratégi cos nessa disputa da formação ética da sociedade diante do trabalho e, con seqü en temente, do modelo de produção e con sumo a que estaremos condicion ados como população, trabalhadores, agentes institucionais, agen tes patronais e intelectuais. A mídia é um dos campos de disputa da informação mais significa tivos assim como todos os processos educativos formais ou informais. Da mesma forma, as empresas através da propaganda e de suas campanhas internas de con trole de atitudes buscam estabelecer padrões de comport am en to e de adesão aos princípios de produção, muitas vezes inadequados aos mecanismos de resistência dos trabalhadores organizados e mesmo incompatíveis com as con cepções e valores deles.

Ao visualizarmos esse arc a bouço celular representativo de processos de vigilância constituídos em rede, observamos que a natureza transversal da ação da VST é algo completamen te evidente. Significa a obviedade da responsabilidade múltipla das instâncias sociais envolvidas nos processos concretos construídos ou a serem construídos a partir das questões apres en tadas pelos também múltiplos objetos. As teias terão características e atores distintos, tan tos quantos forem os obj etos de ação priorizados pelas ações de VST. Nesse processo 
de priorização, devem ser balanceadas as demandas sociais, os impactos epidemiológi cos e as forças existen tes para o exercício das ações decorrentes das disputas e negociações a serem travadas nos vários âmbi tos da célula ou da rede de VST.

\section{Considerações finais}

Esse modelo de redes de VST com suas características heterodoxas e con strutivistas permite a vi sualização do con tex to da ação institucional de VST relacionada às con tradições no âmbi to dos processos de trabalho, bem como das formas em que se or ganizam o Estado e a sociedade para dar conta dos desafios da reprodução social e da sustentabilidade dos modelos de de- senvo lvimento adotados. Con c retamente, é explicitado que a VST é um processo social con t ínuo em que vários atores, inclu sive a sociedade, executam o seu protagonismo cotidianamente em níveis distin tos de ação e com integrações organizadas por processos de promoção da saúde ou de reprodução do capital, em con tex tos perceptíveis e imperceptíveis se tocando continuamente.

Finalmente destacamos o papel das formulações científicas e ideológicas que perm eiam a captu ra ética do que fazer no mu n do do trabalho em sua operação. En contram-se presentes nas disputas dos padrões de pre servação da vida no trabalho a que estão submetidos todos trabalhadores, independentemente do tipo de relação de venda da força de trabalho.

\section{Referências bibliográficas}

Ci rne A 2000. O impa cto da lei estadual sob re as Lesões por Esforços Repetitivos. Dissertação de mestrado. Escola Nacional de Sa ú de Pública, Fiocruz, Rio de Ja n ei ro.

Ech ternacht E 1991. Com quantos paus se faz uma canoa ou com quantas mortes se faz um navio (Sobre o modo de adoecer e morrer dos trabalhadores jatistas em estaleiros do Rio de Janeiro). Dissertação de mestrado. COPPE, Universidade Federal do Rio de Ja n ei ro, Rio de Jan eiro.

Lacaz FAC, Mach ado JHM \& Porto MFS 2002. Es tu do da Situação e Tendências da Vigilância em Saúde do Trabalh ador no Bra sil. Relatório Final [mimeo].

Laurell AC \& Nori ega M 1989. Processo de produção e saúde: trabalho e desgaste operário. Ed. Cebes-Hucitec, São Paulo.

Lima LQ 1997. Al ternativas tecnológicas ao jateamen to de areia seca: a bu sca de inco rporação de novos el ementos na avaliação. Dissertação de mestrado. Escola Nacional de Saú dePública, Fiocruz, Rio de Jan ei ro

Lima L 1993. Construção naval. Dissertação de mestrado. Escola Nacional de Saúde Pública, Fiocruz, Rio de Janeiro.
Mach ado JHM 1996. Alternativas e pro ce ssos de vigilância em saúde do trabalhador: a hetero gen eidade da intervenção. Tese de doutorado. Escola Naci onal de Sa ú de Pública, Fiocruz, Rio de Ja nei ro.

Mach ado JMH, Costa DF, Ca rdoso LM \& Arcu riA 2003. Al ternativas e processos de vigilância em saúde do trabalhador relacion ados à ex posição ao ben zeno no Brasil. Gência e Saúde Col etiva 8(4):913-921.

Porto MFS, Mach ado JMH \& Freitas CM 2000. Promoção da saúde e intersetorialidade: a experiência da Vigilância em Saúde do Trabalhador na Construção de Redes. VI Con gresso Brasileirode Sa ú de Col etiva. Anais. CD-Rom.

Porto MF, Lacaz FAC \& Mach ado JMH 2003. Promoção da Sa ú de e In ters etorialidade: con tri buições e limites da Vigilância em Saúde do Trabalhador no Sistema Único de Sa ú de (SUS). Saúde em Deba te 27(65):192206.

Wisner A 1987. Por den tro do trabalho. Ergonomia: métodos e técnicas. Ed. FTB/Oboré, São Paulo.

Artigo apresen t ado em 20/07/2005

Aprovado em 31/08/2005

Versão final apresen tada em 5/09/2005 